\title{
30 A CASE STUDY: ACTIVE LEARNING FROM A \\ PROGRAMME TO REDUCE INJURY WHERE ALCOHOL IS A CONTRIBUTING FACTOR
}

doi:10.1136/injuryprev-2012-040580d.30

K Harper*. Accident Compensation Corporation, Programme Manager, Vogel Centre, Wellington, New Zealand

New Zealand recognises it has a drinking culture that affects the quality of life for both drinkers and non-drinkers. This affect is most often associated with health and social impacts. However, it is estimated that $22 \%$ of Accident Compensation claims, under the ACC scheme, are alcohol related and cost $\$ 650$ million per year.

This indifference toward injury is due, in part at least, to public attitude towards alcohol and the policies that affect this. As a result, preventing alcohol-related injury is dependent on understanding the complex interplay between individual, community and societal factors.

The goal of the ACC alcohol-related injury programme is to reduce the cost of injury where alcohol is a contributing factor. This requires a broad approach, combining public health and injury prevention practices. As such, there is a need to balance short and medium term efforts to reduce the risk of alcohol contributing to injury and long-term objectives to prevent alcohol as a cause of poor health.

The programme strategy aims to synchronise education, engineering and enforcement based intervention with audience contemplation, determination and action. Through this approach the programme forecasts a return on investment for injury prevention and longer-term impact on alcohol-related health and social issues.

This case study reports active learning from the programme design and development and applies these to ongoing interventions. 